\title{
Cost Comparison of Peri-Strips Dry®/Peri-Strips Dry with Veritas $\circledR$ (PSD/PSD-V) Versus Seamguard $\AA$ in Gastric Staple Line Reinforcement (SLR)
}

\author{
Manuel G Ramirez ${ }^{* 1}$, Alex Gandsas ${ }^{2}$, Barbara Blaylock ${ }^{3}$, Josh Epstein ${ }^{3}$ and Manuel A Ramirez ${ }^{4}$ \\ ${ }^{1}$ Baxter Healthcare Corporation, Deerfield, USA
}

${ }^{2}$ Anne Arundel Medical Center, Annapolis, USA

${ }^{3}$ Stratevi, Santa Monica, USA

${ }^{4}$ Baxter México, Chapultepec Morales, México

*Corresponding author: Manuel G Ramirez, Global Advanced Surgery Clinical/HEOR, Advanced Surgery, Baxter Healthcare Corporation, 1 Baxter Way, Deerfield, IL 60015, USA.

To Cite This Article: Manuel G Ramirez. Cost Comparison of Peri-Strips Dry®/Peri-Strips Dry with Veritas® (PSD/PSD-V) Versus Seamguard® in Gastric Staple Line Reinforcement (SLR). Am J Biomed Sci \& Res. 2019 - 6(1). AJBSR.MS.ID.000998. DOI: 10.34297/AJBSR.2019.06.000998.

Received: 眥 October 18, 2016; Published: 踚 November 05, 2019

\begin{abstract}
Background: A recent meta-analysis of staple line complications (SLC) in primary bariatric/metabolic procedures showed significantly lower $(\mathrm{p}<0.05)$ SLC rates for bovine pericardium (Peri-Strips Dry/Peri-Strips Dry with Veritas (PSD/PSD-V); Baxter Healthcare, Deerfield, IL) compared to biocompatible glycolide copolymer buttress (Seamguard; W.L. Gore \& Associates, Inc, Flagstaff, AZ), suture reinforcement (oversewing), and no reinforcement.
\end{abstract}

Objective: The aim of this study is to assess the impact of staple line reinforcement (SLR) choice on costs and clinical outcomes of primary bariatric/ metabolic procedures from the hospital administrator perspective in four countries - the United States (US), Brazil, Colombia, and Mexico.

Methods: The cost-consequence model assesses PSD/PSD-V vs. Seamguard, oversewing, or no SLR. Epidemiologic inputs are from the literature. Clinical inputs are from the literature (SLC rates and operating room (OR) time per procedure) or are based on US databases (US SLC rates, product volume per procedure). Product costs are US average sales price. Other cost inputs (complications, OR time and oversewing) are from government sources. All costs are reported in 2018 country currencies.One-way sensitivity analyses using $95 \%$ or $+/-20 \%$ when the confidence interval was not available and probabilistic sensitivity analyses (PSA) using Monte Carlo simulation for 5000 iterations evaluate key drivers of costs.

Results: Analysis was completed based on hospitals conducting 100 primary bariatric/metabolic procedures annually. Reinforcement of gastric staple lines with PSD/PSD-V vs current approach mix would lead to fewer complications and reduction in surgical time leading to net cost savings. PSD/PSD-V remains a dominant strategy (fewer complications, lower costs) in the PSA. Net annual cost savings would be US\$27,537, R $\$ 152,399$, COL\$139.81 million and Mex\$1,854,032, for US, Brazil, Colombia and Mexico respectively.

Conclusion: Hospital administrators can expect cost savings associated with decreased complications and OR time equivalent to or up to 2 times greater than the additional product acquisition cost when PSD/PSD-V is used for gastric SLR.

Keywords: Peri-Strips Dry; Seamguard; Staple line reinforcement; Gastric bypass; Sleeve gastrectomy; Cost

Abbreviations: BMI: Body Mass Index; COL\$: Columbian Pesos; IMSS: Mexican Social Security Institute; ICU: Intensive Care Unit; Mex\$: Mexican Pesos; OR: Operating Room; PSA: Probabilistic Sensitivity Analysis; PSD/PSD-V: Peri-Strips Dry/Peri-Strips Dry with Veritas; R\$: Brazilian Reals; SLC: Staple Line Complications; SLR: Staple Line Reinforcement; US: United States; US\$: US Dollars

\section{Introduction}

\section{Increasing need for primary bariatric/metabolic surgery in Latin America}

The prevalence of morbid obesity (body mass index (BMI) > $=40$ $\mathrm{kg} / \mathrm{m}^{2}$ ) is highest among men and women in high-income western countries (men=3.3\%; women=5.7\%) [1]. Low- and middle-income countries such as those in Latin America have lower prevalence, but the rate of morbid obesity among adults is increasing rapidly [1]. In the last decade (2007-2016), prevalence of morbid obesity among adult men and women in Latin America has approximately doubled (2.1 and 1.5 times) to $1.2 \%$ and $3.2 \%$, respectively [1]. 
Bariatric surgery is indicated to manage morbid obesity, severe obesity (BMI=35-39.9 kg/m2) with obesity-related comorbidities, and obesity (30-34.9 kg/m2) with difficult to control comorbidities [2]. The American Society for Metabolic and Bariatric Surgery (ASMBS) has favorably compared the most common bariatric surgery procedures - Roux-en-Y gastric bypass (gold standard) and sleeve gastrectomy - for management of weight and comorbidities [3]. Sleeve gastrectomy has overtaken Roux-en-Y gastric bypass as the most common primary bariatric/metabolic procedure worldwide [4]. However, the most common surgery type in many Latin American countries continues to be Roux-en-Y gastric bypass by a wide margin [5].

\section{High cost of staple line complications (SLC)}

According to the annual report on outcomes after bariatric surgery published by the International Federation for the Surgery of Obesity and Metabolic Disorders (IFSO), most primary bariatric/metabolic procedures are conducted laparoscopically worldwide (99.3\%) with a good safety profile [5]. However, staple line complications (SLC), such as hemorrhage (weighted average across procedures $=2.76 \%$ ) and leak (weighted average across procedures $=2.46 \%$ ) may be severe [6]. Multiple studies have examined the healthcare resource utilization and expenditures associated with the correction of hemorrhages and leaks after primary bariatric surgery with an average cost of leaks after sleeve gastrectomy between US\$22,014-\$93,451[7-10]. The largest contributors of expense included room and board on the ward and intensive care unit (ICU) (The Netherlands: 82\%; US: 44\%), therapeutic measures such as reintervention (The Netherlands: 11\%; US: $24 \%$ ), and diagnostic measures such as imaging (The Netherlands: 8\%; US: 14\%) [7, 8].

\section{Reduce SLC using Peri-Strips Dry with Veritas (PSD-V) for staple line reinforcement (SLR)}

Staple line reinforcement (SLR) is the reinforcing or buttressing of the gastric staple line with the goal of reducing SLC following primary bariatric/metabolic surgery. There is no standard of care (SOC) for gastric SLR [11]. In fact, during many primary bariatric/ metabolic surgeries no SLR is administered, likely due to perceived cost [6]. To fill this gap in care, multiple products and methods have been deployed for SLR. Peri-Strips Dry ${ }^{\circledR}$ with Veritas ${ }^{\circledR}$ Collagen Matrix (PSD-V; Baxter Healthcare, Deerfield, IL) is prepared from dehydrated bovine pericardium procured from cattle under 30 months of age in the United States [12]. PSD-V was preceded by Peri-Strips Dry® Bovine Pericardium Strips (PSD; Baxter Healthcare, Deerfield, IL) [13]. Seamguard (W.L. Gore \& Associates, Inc, Flagstaff, AZ) is another commonly used buttressing option made from synthetic biocompatible glycolide copolymer [14]. Another method of gastric SLR is oversewing of the suture line; however, this is associated with increased OR time.

The American Society for Metabolic and Bariatric Surgery (ASMBS) database of bariatric surgeries shows low rates $(<1 \%)$ of leaks and bleeds for both sleeve gastrectomy and Roux-en-Y gastric bypass in the US [3]. A recent meta-analysis by Shikora et al. (2015) based on 253 studies covering 66,727 unique patients favorably compared PSD/PSD-V to Seamguard, oversewing, and no SLR for primary gastric bypass and sleeve gastrectomy surgeries [6]. Overall, the use of PSD/PSD-V for gastric SLR was associated with a significant reduction in SLC including both leaks and bleeds after both gastric bypass and sleeve gastrectomy [6]. The aim of this study is to apply costs to those results and assess the impact of SLR choice on costs and outcomes of primary gastric bypass and sleeve gastrectomy from the hospital administrator perspective in the US, Brazil, Colombia, and Mexico.

\section{Materials and Methods}

A cost-consequence model was developed to compare the cost impact associated with using PSD/PSD-V for SLR in primary bariatric/metabolic surgery as compared to other SLR methods including Seamguard, oversewing, and no reinforcement. Costs from the hospital administrator perspective include the difference in the distribution of surgeries, rates of SLC including leaks and bleeds, surgery time, and product costs. Four countries were selected based on geographic region (Americas) and availability of country-specific data: the US, Brazil, Colombia, and Mexico. Epidemiologic, clinical, and cost inputs are shown in Table 1 (by SLR method) and Table 2 (by country).

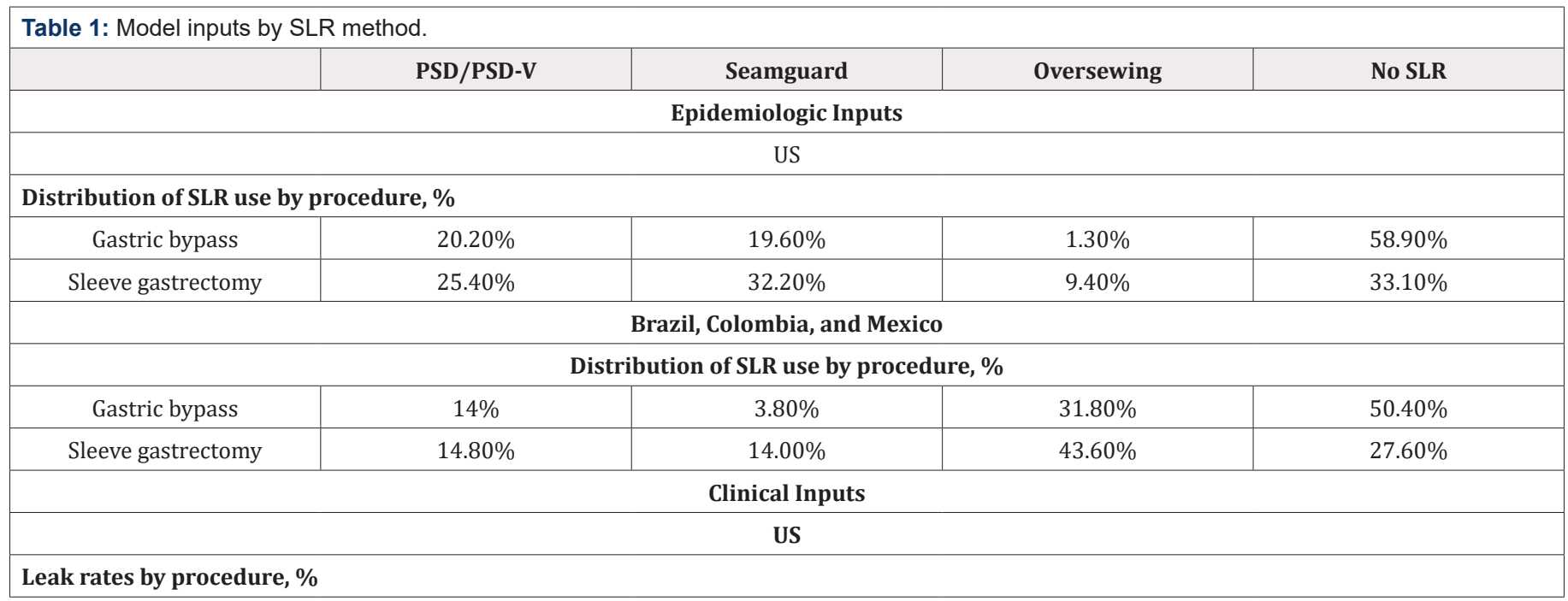




\begin{tabular}{|c|c|c|c|c|}
\hline Gastric bypass & $0.16 \%$ & $0.31 \%$ & $0.40 \%$ & $0.42 \%$ \\
\hline Sleeve gastrectomy & $0.14 \%$ & $0.25 \%$ & $0.21 \%$ & $0.25 \%$ \\
\hline \multicolumn{5}{|c|}{ Bleed rates by procedure, $\%$} \\
\hline Gastric bypass & $0.41 \%$ & $0.96 \%$ & $0.98 \%$ & $0.99 \%$ \\
\hline Sleeve gastrectomy & $0.12 \%$ & $0.21 \%$ & $0.25 \%$ & $0.51 \%$ \\
\hline \multicolumn{5}{|c|}{ Brazil, Colombia, and Mexico } \\
\hline \multicolumn{5}{|c|}{ Leak rates by procedure, $\%$} \\
\hline Gastric bypass & $1.00 \%$ & $1.88 \%$ & $2.44 \%$ & $2.60 \%$ \\
\hline Sleeve gastrectomy & $1.83 \%$ & $3.25 \%$ & $2.70 \%$ & $3.27 \%$ \\
\hline \multicolumn{5}{|c|}{ Bleed rates by procedure, $\%$} \\
\hline Gastric bypass & $1.28 \%$ & $3.02 \%$ & $3.10 \%$ & $3.13 \%$ \\
\hline Sleeve gastrectomy & $1.16 \%$ & $2.09 \%$ & $2.41 \%$ & $4.94 \%$ \\
\hline \multicolumn{5}{|l|}{ Units per procedure } \\
\hline Gastric bypass & 3.7 & 2.4 & 24 & -- \\
\hline Sleeve gastrectomy & 5.2 & 5.2 & 33 & -- \\
\hline \multicolumn{5}{|c|}{ Operating room time by procedure, minutes } \\
\hline Gastric bypass & 120 & 124 & 229 & 186 \\
\hline Sleeve gastrectomy & 59 & 61 & 73 & 59 \\
\hline
\end{tabular}

Abbreviations: PSD/PSD-V: Peri-Strips Dry/Peri-Strips Dry with Veritas; SLR: Suture Line Reinforcement

\begin{tabular}{|c|c|c|c|c|}
\hline & United States & Brazil & Colombia & Mexico \\
\hline \multicolumn{5}{|c|}{ Epidemiologic Inputs } \\
\hline \multicolumn{5}{|c|}{ Distribution of primary bariatric procedures, $\%$} \\
\hline Gastric bypass & $41 \%$ & $92 \%$ & $72 \%$ & $86 \%$ \\
\hline Sleeve gastrectomy & $59 \%$ & $8 \%$ & $28 \%$ & $14 \%$ \\
\hline \multicolumn{5}{|c|}{ Cost Inputs } \\
\hline \multicolumn{5}{|c|}{ Product costs } \\
\hline PSD/PSD-V, per firing & US\$166 & $\mathrm{R} \$ 611$ & COL $\$ 493,865$ & Mex\$3,199 \\
\hline Seamguard, per firing & US $\$ 167$ & $\mathrm{R} \$ 613$ & COL $\$ 495,378$ & Mex $\$ 3,209$ \\
\hline \multicolumn{5}{|l|}{ Oversewing, per piece } \\
\hline Gastric bypass & US\$16 & $\mathrm{R} \$ 59$ & $\operatorname{COL} \$ 47,377$ & Mex\$307 \\
\hline Sleeve gastrectomy & US $\$ 5$ & $\mathrm{R} \$ 19$ & COL $\$ 15,115$ & Mex $\$ 98$ \\
\hline \multicolumn{5}{|l|}{$\begin{array}{l}\text { Cost of complications per } \\
\text { procedure }\end{array}$} \\
\hline \multicolumn{5}{|l|}{ Gastric bypass } \\
\hline Leaks & US $\$ 25,010$ & $\mathrm{R} \$ 8,205$ & $\operatorname{COL} \$ 35,368,824$ & Mex $\$ 480,613$ \\
\hline Bleeds & US $\$ 26,036$ & $\mathrm{R} \$ 12,343$ & $\operatorname{COL} \$ 38,679,096$ & $\operatorname{Mex} \$ 500,317$ \\
\hline \multicolumn{5}{|l|}{ Sleeve gastrectomy } \\
\hline Leaks & US $\$ 12,381$ & $\mathrm{R} \$ 3,981$ & COL $\$ 17,672,244$ & Mex $\$ 237,915$ \\
\hline Bleeds & US $\$ 13,406$ & $\mathrm{R} \$ 8,119$ & COL $\$ 20,982,516$ & Mex $\$ 257,619$ \\
\hline $\begin{array}{l}\text { Cost of operating room } \\
\text { time, per hour }\end{array}$ & US $\$ 1,875$ & $\mathrm{R} \$ 2,593$ & COL $\$ 2,094,032$ & $\operatorname{Mex} \$ 13,566$ \\
\hline
\end{tabular}

Abbreviations: COL\$, Colombian Pesos; Mex\$, Mexican Pesos; PSD/PSD-V, Peri-Strips Dry/Peri-Strips Dry with Veritas; R\$, Brazilian Reals; US\$, United States dollars.

\section{Epidemiologic Inputs}

Country-specific rates of gastric bypass and sleeve gastrectomy are from the international survey of primary bariatric/metabolic performed annually by IFSO [15]. The rates of gastric bypass and sleeve gastrectomy data for Brazil were supplemented with a report by the Brazilian Ministry of Health [16]. The rates of gastric bypass and sleeve gastrectomy data for the US were supplemented with 
data from US databases $[17,18]$. The proportions of procedures using each type of SLR - PSD/PSD-V, Seamguard, oversewing, and no SLR - are from the meta-analysis by Shikora et al. (2015) [6] for Latin American countries with supplemental information from US databases for the US [17-19]. As bariatric surgical centers are often specialized, annual outcomes are reported based on a high-volume hospital performing 100 surgeries per year [20,21].

\section{Clinical Inputs}

The rate of SLC including leaks \& bleed following gastric bypass and sleeve gastrectomy by SLR method are from the meta-analysis by Shikora et al. (2015) [6] for Latin American countries with supplemental information from US databases for the US [17-19]. The number of firings per procedure for PSD-V and Seamguard were calculated from the Premier US Data Base 2017 [22]. The supplies for oversewing are based on supplies for the diagnosis related groups (DRG) for sleeve gastrectomy with complications (DRG=619) and gastric bypass with complications (DRG=620) from the Mexican Social Security Institute (IMSS) 2018 [23,24]. Operating room time for gastric bypass and sleeve gastrectomy by SLR method were taken from the literature [25-27]. Because the length of surgery for gastric bypass with oversewing SLR could not be identified, the same percentage increase $(24 \%)$ in the length of surgery for sleeve gastrectomy with oversewing versus with no SLR was applied [26].

\section{Cost Inputs}

Product Costs: Given lack of visibility to product costs in each country, costs for PSD-V and Seamguard are based on the US average sales price (ASP) in 2018 [28]. Product and all other costs were exchanged and inflated to 2018 currency using the exchange rates and country-specific consumer price index (CPI) published by the International Monetary Fund (IMF) [29,30].

Cost of Complications: The associated cost of a SLC (leak or bleed) was applied to the specific surgery type. For bleed complications, the additional cost of a blood bank event ( 6 units) was added [31]. Costs of complications following sleeve gastrectomy for Brazil, Colombia, Mexico, and the US were calculated based on DRG 619 from the Mexican Social Security Institute Inpatient Hospital DRG's Book (IMSS) 2016 including sutures (34 units), hospital length of stay (LOS; 12 days), and ICU LOS (4 days) [23]. Costs of complications following gastric bypass for Brazil, Colombia, Mexico, and the US were calculated based on DRG 620 from the IMSS 2016 including sutures (24 units), hospital LOS (24.5 days), and ICU LOS (8 days) [24]. Costs of hospital and ICU room and board are all country specific.

Cost of Operating Room Time: Cost of operating room (OR) time per hour for Brazil, Colombia and Mexico is based on IMSS 2017 [32]. Whereas, cost of US OR time was calculated based on national average of OR cost per minute across five surgical procedures (laparoscopic prostatectomy, myringotomy with insertion of tube, laparoscopic inguinal hernia repair, bilateral reduction mammoplasty, and knee arthroscopy) and surgical specialties from a university hospital perspective [33].

\section{Sensitivity Analysis}

Sensitivity analyses were conducted for both annual net cost impact results and net cost impact results per procedure. Oneway sensitivity analyses used the $95 \%$ confidence intervals or $+/$ $20 \%$ when the confidence interval was not available. Probabilistic sensitivity analyses (PSA) using Monte Carlo simulation for 5000 iterations evaluated key drivers of costs. The distribution of SLR use by procedure type was varied jointly. Distributions of variables for the PSA are included in the Appendix. The 95\% confidence interval (CI) is reported for net annual cost impact and cost impact per procedure from the PSA results.

\section{Results and Discussion}

\section{Results}

Overall: For hospitals conducting 100 primary bariatric/metabolic procedures annually, the model estimated the model estimated avoidance of 2 complications and a reduction in hours of surgical time (71 US; 105 Brazil; 84 Colombia, 99 Mexico) (Table 3) by using PSD/PSD-V in all surgeries. Net annual cost savings from hospital resource utilization would be US\$27,537 (95\% CI=US\$6,572US\$51,509), $\quad \mathrm{R} \$ 152,399 \quad(95 \% \quad \mathrm{CI}=\mathrm{R} \$ 66,764-\mathrm{R} \$ 253,925)$, COL $\$ 139.81$ million $(95 \%$ CI=COL $\$ 72.48$ million-COL $\$ 220.95$ million), and Mex\$1,854,032 (95\% CI=Mex\$1,256,153Mex $\$ 2,560,758$ ) (Figure 1).

Table 3: Estimated annualized clinical impact of using PSD/PSD-V.

\begin{tabular}{|c|c|c|c|c|}
\hline & United States & Brazil & Colombia & Mexico \\
\hline \multicolumn{5}{|l|}{ Overall } \\
\hline \multicolumn{5}{|l|}{ Complications Avoided } \\
\hline Leaks & 0.12 & 1.27 & 1.21 & 1.25 \\
\hline Bleeds & 0.29 & 1.59 & 1.62 & 1.6 \\
\hline Operating Room Savings, hours & 71 & 104.9 & 84.5 & 98.7 \\
\hline \multicolumn{5}{|l|}{ Seamguard vs. PSD/PSD-V } \\
\hline Procedures, N & 27.1 & 4.6 & 6.7 & 5.2 \\
\hline \multicolumn{5}{|l|}{ Complications Avoided } \\
\hline Leaks & 0.03 & 0.05 & 0.08 & 0.06 \\
\hline Bleeds & 0.06 & 0.07 & 0.08 & 0.08 \\
\hline Operating Room Savings, hours & 1.2 & 0.2 & 0.3 & 0.3 \\
\hline
\end{tabular}




\begin{tabular}{|c|c|c|c|c|}
\hline \multicolumn{5}{|l|}{ Oversewing vs. PSD/PSD-V } \\
\hline Procedures, N & 6.1 & 32.7 & 35.1 & 33.5 \\
\hline \multicolumn{5}{|l|}{ Complications Avoided } \\
\hline Leaks & 0.01 & 0.45 & 0.44 & 0.45 \\
\hline Bleeds & 0.01 & 0.58 & 0.57 & 0.57 \\
\hline Operating Room Savings, hours & 2.3 & 54 & 44.5 & 51.1 \\
\hline \multicolumn{5}{|l|}{ No SLR vs. PSD/PSD-V } \\
\hline Procedures, N & 43.6 & 48.6 & 44 & 47.2 \\
\hline \multicolumn{5}{|l|}{ Complications Avoided } \\
\hline Leaks & 0.08 & 0.77 & 0.69 & 0.75 \\
\hline Bleeds & 0.22 & 0.94 & 0.96 & 0.95 \\
\hline Operating Room Savings, hours & 26.4 & 50.6 & 39.7 & 47.3 \\
\hline
\end{tabular}

Abbreviations: PSD/PSD-V: Peri-Strips Dry/Peri-Strips Dry with Veritas.

(Figure 2) shows the influence of cost drivers on the overall annual net cost savings. The top five cost drivers across countries (listed by aggregate rank) included the cost per firing of PSD/ PSD-V (US: 1; Brazil: 2; Colombia: 1; Mexico: 2), the proportion of gastric bypass procedures (US: rank $=2$; Brazil: rank=1; Colombia:

4; Mexico: 6), the annual number of bariatric/metabolic procedures (US: 7; Brazil: 4; Colombia: 2; Mexico: 1), the number of firings of PSD/PSD-V during gastric bypass (US: 8; Brazil: 3; Colombia: 3; Mexico: 3), and the OR time difference in gastric bypass surgery with no SLR (US: 3; Brazil: 6; Colombia: 6; Mexico: 5).

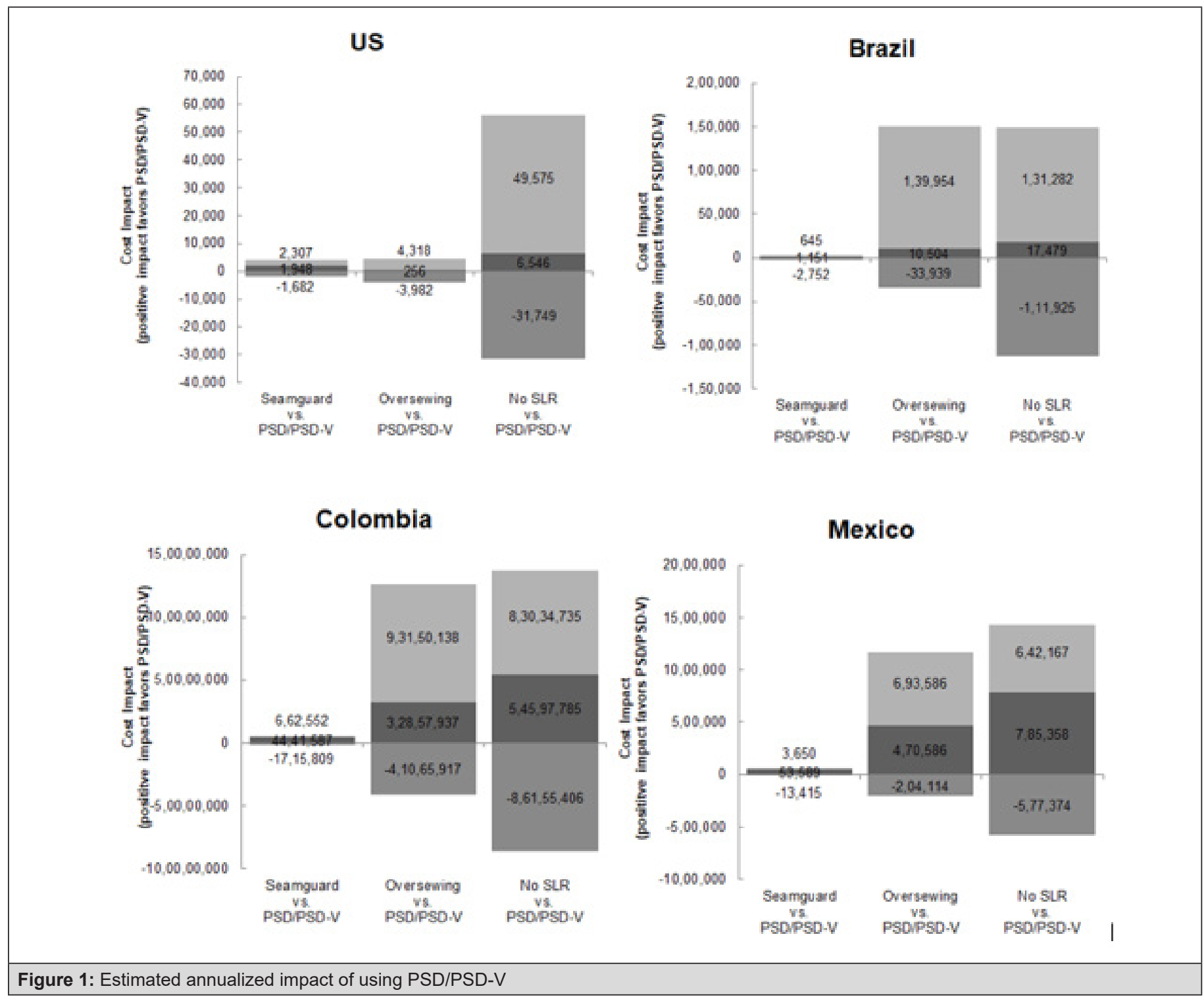


US

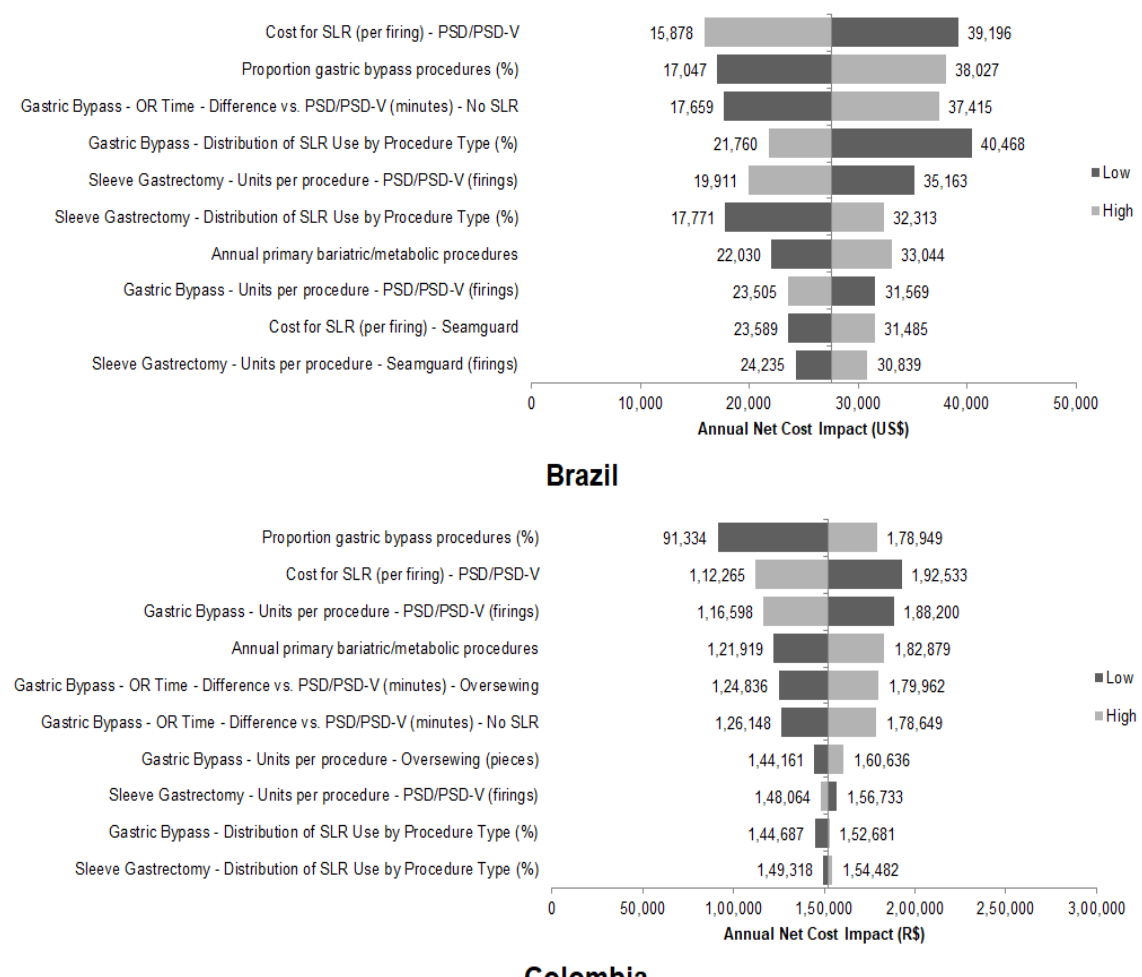

Colombia

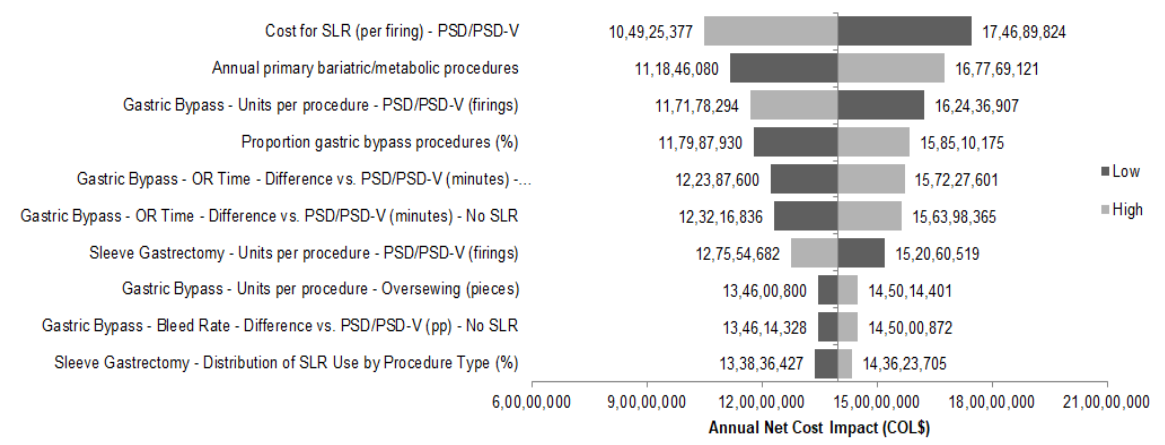

Mexico

Annual primary bariatric/metabolic procedures

Cost for SLR (per firing) - PSD/PSD-V

Gastric Bypass - Units per procedure - PSD/PSD-V (firings) Gastric Bypass - OR Time - Difference vs. PSD/PSD-V (minutes) - Oversewing

Gastric Bypass - OR Time - Difference vs. PSD/PSD-V (minutes) - No SLR Proportion gastric bypass procedures (\%)

Gastric Bypass - Distribution of SLR Use by Procedure Type (\%) Gastric Bypass - Bleed Rate - Difference vs. PSD/PSD-V (pp) - No SLR Gastric Bypass - Leak Rate - Difference vs. PSDIPSD-V (pp) - No SLR Gastric Bypass - Bleed Rate - Difference vs. PSD/PSD-V (pp) - Oversewing

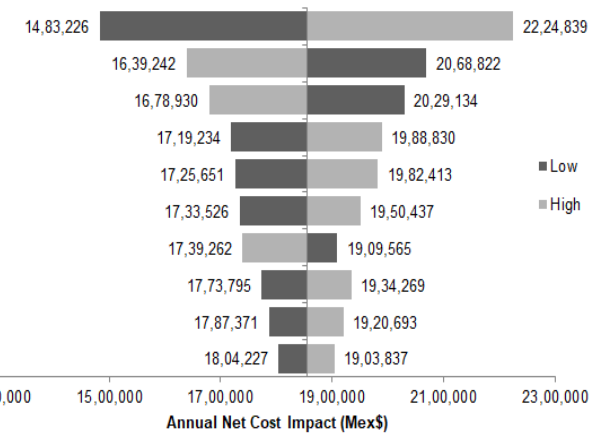

Figure 2: Estimated annualized impact of using PSD/PSD-V - One Way Sensitivity Analysis - Tornado Diagram

Seamguard vs. PSD/PSD-V: Based on the distributions of primary bariatric/metabolic procedures and SLR choice for those procedures, the estimated number of annual primary bariatric/ metabolic procedures performed using Seamguard for SLR would be $27,5,7$, and 5 per 100 bariatric/metabolic surgeries in the
US, Brazil, Colombia, and Mexico, respectively. Switching from Seamguard to PSD/PSD-V only represents $1-9 \%$ of the overall annual net cost impact.

Per procedure, switching to PSD/PSD-V from Seamguard could provide net savings of US\$95 (95\% CI=-US\$186-US\$376) 
and Mex\$8,382 (95\% CI=Mex\$3,762-Mex\$12,913). In Brazil and Colombia, the net impact of switching to PSD/PSD-V from Seamguard is not significantly different than zero $(-\mathrm{R} \$ 207,95 \%$ $\mathrm{CI}=-\mathrm{R} \$ 985-\mathrm{R} \$ 567$; COL\$0.51 million, 95\% CI=-COL\$0.27 millionCOL\$1.29 million). Although the use of Seamguard is associated with fewer firings per procedure, the costs savings from using PSD/ PSD-V vs. Seamguard from reduced complications and OR time are greater than or approximately equivalent to the increase in product acquisition costs (Figure 1).

Influence of cost drivers per procedure savings from PSD/
PSD-V vs Seamguard were identified across countries (Figure 3). Top five cost drivers across countries (listed by aggregate rank) included the following: cost per firing of PSD/PSD-V (US: rank=1; Brazil: 2; Colombia: 1; Mexico: 2), the cost per firing of Seamguard (US: 2; Brazil: 3; Colombia: 2; Mexico: 3), the number of firings of PSD/PSD-V during gastric bypass (US: 5; Brazil: 4; Colombia: 5; Mexico: 4), the number of firings of Seamguard during sleeve gastrectomy (US: 3; Brazil: 7; Colombia: 3; Mexico: 5), and the number of firings of PSD/PSD-V during sleeve gastrectomy (US: 4; Brazil: 8; Colombia: 4; Mexico: 6).

US

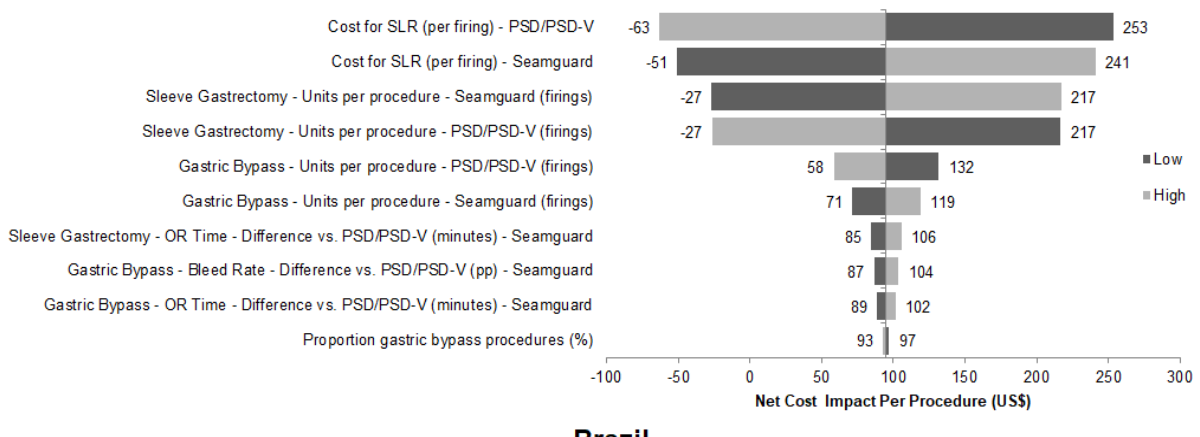

Brazil
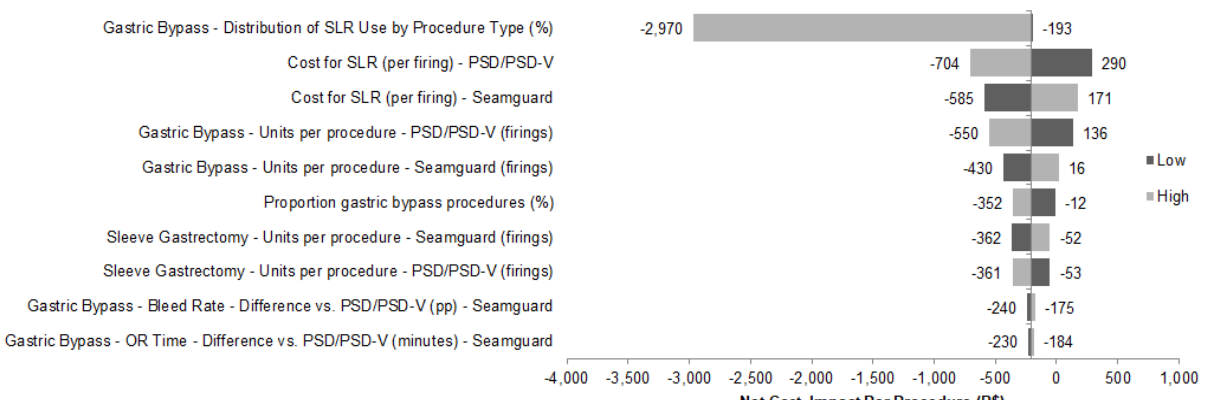

$\begin{array}{rrrr}-4,000 & -3,500 & -3,000 & -2,50 \\ & \text { Net Cost Impact Per Procedure (RS) }\end{array}$

Colombia

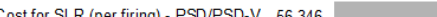

Cost for SLR (per firing) - PSD/PSD-V 56,346 Cost for SLR (per firing) - Seamguard Sleeve Gastrectomy - Units per procedure - Seamguard (firings) Sleeve Gastrectomy - Units per procedure - PSD/PSD-V (firings) Gastric Bypass - Units per procedure - PSD/PSD-V (firings) Gastric Bypass - Units per procedure - Seamguard (firings) Gastric Bypass - Bleed Rate - Difference vs. PSD/PSD-V (pp) - Seamguard Sleeve Gastrectomy - Leak Rate - Difference vs. PSD/PSD-V (pp) - Seamguard Gastric Bypass - Distribution of SLR Use by Procedure Type (\%) Gastric Bypass - Leak Rate - Difference vs. PSDIPSD-V (pp) - Seamguard

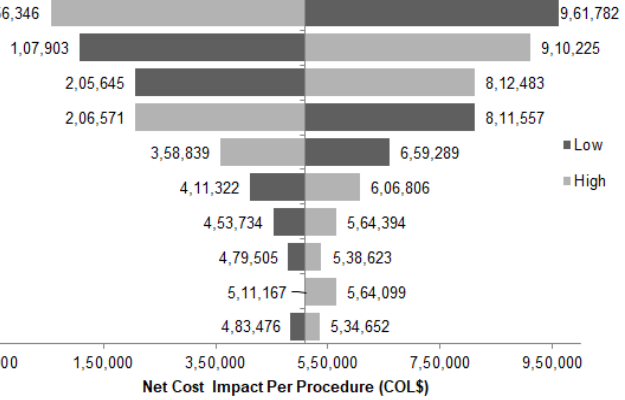

\section{Mexico}

Gastric Bypass - Distribution of SLR Use by Procedure Type (\%) Cost for SLR (per firing) - PSD/PSD-V Cost for SLR (per firing) - Seamguard Gastric Bypass - Units per procedure - PSD/PSD-V (firings) Sleeve Gastrectomy - Units per procedure - Seamguard (firings) Sleeve Gastrectomy - Units per procedure - PSD/PSD-V (firings) Gastric Bypass - Bleed Rate - Difference vs. PSD/PSD-V (pp) - Seamguard Gastric Bypass - Units per procedure - Seamguard (firings) Gastric Bypass - Leak Rate - Difference vs. PSDIPSD-V (pp) - Seamguard Proportion gastric bypass procedures $(\%)$

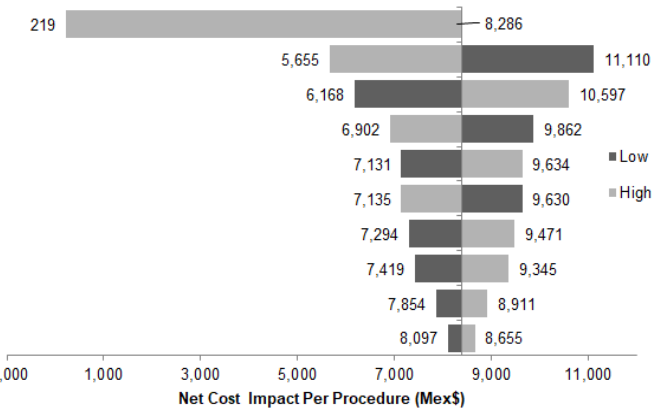

Figure 3: Estimated impact of using Seamguard vs. PSD/PSD-V per procedure - One Way Sensitivity Analysis - Tornado Diagram 
Oversewing vs. PSD/PSD-V: Based on the distributions of primary bariatric/metabolic procedures and SLR choice for those procedures, the estimated number of annual primary bariatric/ metabolic procedures performed using oversewing for SLR would be $6,33,35$, and 33 per 100 bariatric/metabolic surgeries in the US, Brazil, Colombia, and Mexico, respectively. Switching from oversewing to PSD/PSD-V represents over half (52-76\%) of the overall annual net cost impact in Latin American countries.

Higher product acquisition costs associated with PSD/PSD-V vs. oversewing are less than the cost savings associated with reduced complications and OR time (Figure 1). Per procedure, switching to PSD/PSD-V from oversewing could provide net savings of US $\$ 98$ (95\% CI=-US\$188-US\$391), R \$3,558 (95\% CI=R \$2,199-R \$5,047),
COL $\$ 2.42$ million (95\% CI=COL $\$ 1.47$ million-COL $\$ 3.51$ million), Mex $\$ 28,700$ (95\% CI=Mex $\$ 21,592$-Mex $\$ 37,049)$.

The top five cost drivers of the per procedure savings from PSD/PSD-V vs. oversewing (listed by aggregate rank) included the cost per firing of PSD/PSD-V (US: rank=3; Brazil: 3; Colombia: 2; Mexico: 2), the OR time difference in gastric bypass surgery for oversewing (US: 7; Brazil: 2; Colombia: 1; Mexico: 1), the proportion of gastric bypass procedures (US: 5; Brazil: 1; Colombia: 3; Mexico: 3 ), the number of firings of PSD/PSD-V during gastric bypass (US: 9; Brazil: 4; Colombia: 4; Mexico: 4), and the number of firings of PSD/PSD-V during sleeve gastrectomy (US: 4; Brazil: 6; Colombia: 5; Mexico: 8) (Figure 4).

US

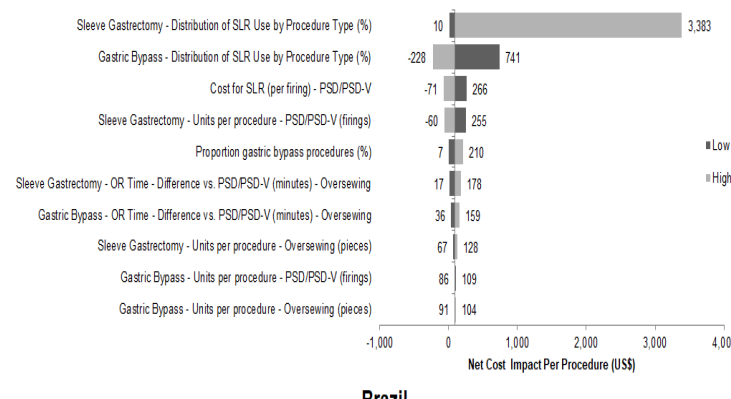

Brazil

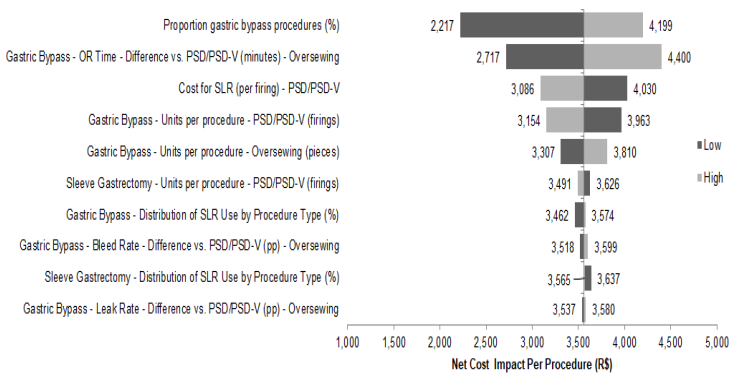

Colombia

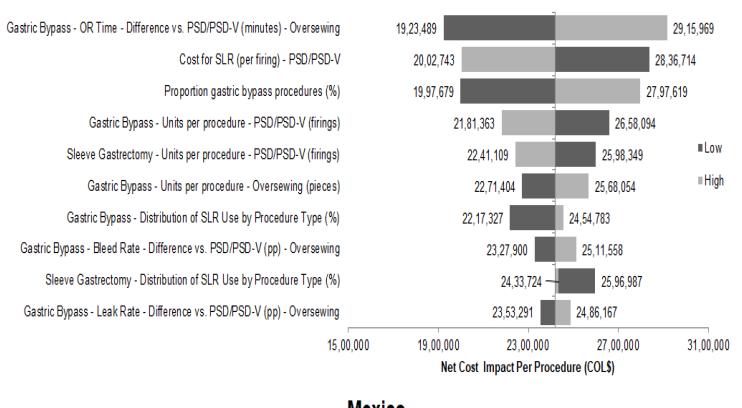

Mexico

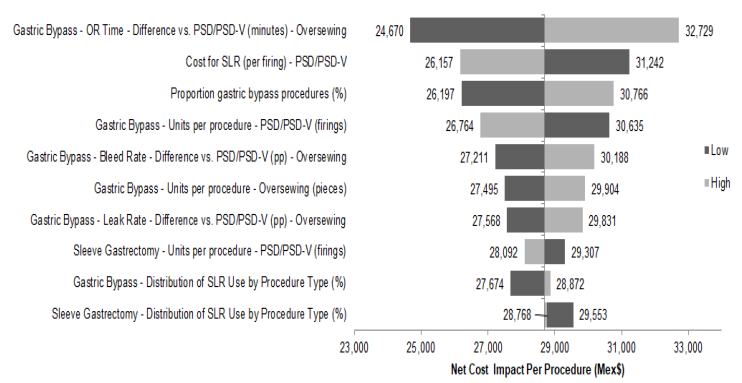

Figure 4. Estimated impact of using No SLR vs. PSD/PSD-V per procedure - One Way Sensitivity Analysis - Tornado Diagram. 
No SLR vs. PSD/PSD-V: No utilization of SLR in primary bariatric/ metabolic procedures performed is estimated to be $44,49,44$, and 47 per 100 annual procedures in the US, Brazil, Colombia, and Mexico, respectively. Switching to PSD/PSD-V from no SLR is associated with the highest reductions in annual complications (US: 0.3, 73\%; Brazil: 1.7, 60\%; Colombia: 1.7, 59\%; Mexico: 1.7, 60\%;). Implementation of PSD/PSD-V vs. no SLR represents cost savings of approximately $24 \%-89 \%$ of the overall annual net cost impact.

While there is a higher product acquisition cost associated with PSD/PSDV vs no SLR, it is less than or approximately equivalent to the cost savings associated with reduction in complications and OR time (Figure 1).

Implementation of PSD/PSDV vs no SLR could provide net savings per procedure of US\$559 (95\% CI=US\$212-US\$949),
COL\$1.17 million (95\% CI=COL $\$ 0.42$ million-COL $\$ 1.98$ million), and Mex\$18,009 (95\% CI=Mex\$12,145 -Mex\$24,311). In Brazil, the net impact of switching to PSD/PSD-V from no SLR is not significantly different than zero $(\mathrm{R} \$ 758,95 \% \mathrm{CI}=-\mathrm{R} \$ 197-\mathrm{R} \$ 1,775)$.

Figure 5 shows the influence of cost drivers on the per procedure savings from PSD/PSD-V vs. no SLR. The top five drivers of cost (listed by aggregate rank) included the OR time difference in gastric bypass surgery for no SLR (US: rank=1; Brazil: 1; Colombia: 2; Mexico: 1), the cost per firing of PSD/PSD-V (US: 4; Brazil: 2; Colombia: 1; Mexico: 2), the number of firings of PSD/PSD-V during gastric bypass (US: 7; Brazil: 3; Colombia: 3; Mexico: 3), the distribution of SLR choice in sleeve gastrectomy surgery (US: 2; Brazil: 5; Colombia: 4; Mexico: 6), and the proportion of gastric bypass procedures (US: 3; Brazil: 4; Colombia: 5; Mexico: 7).

US
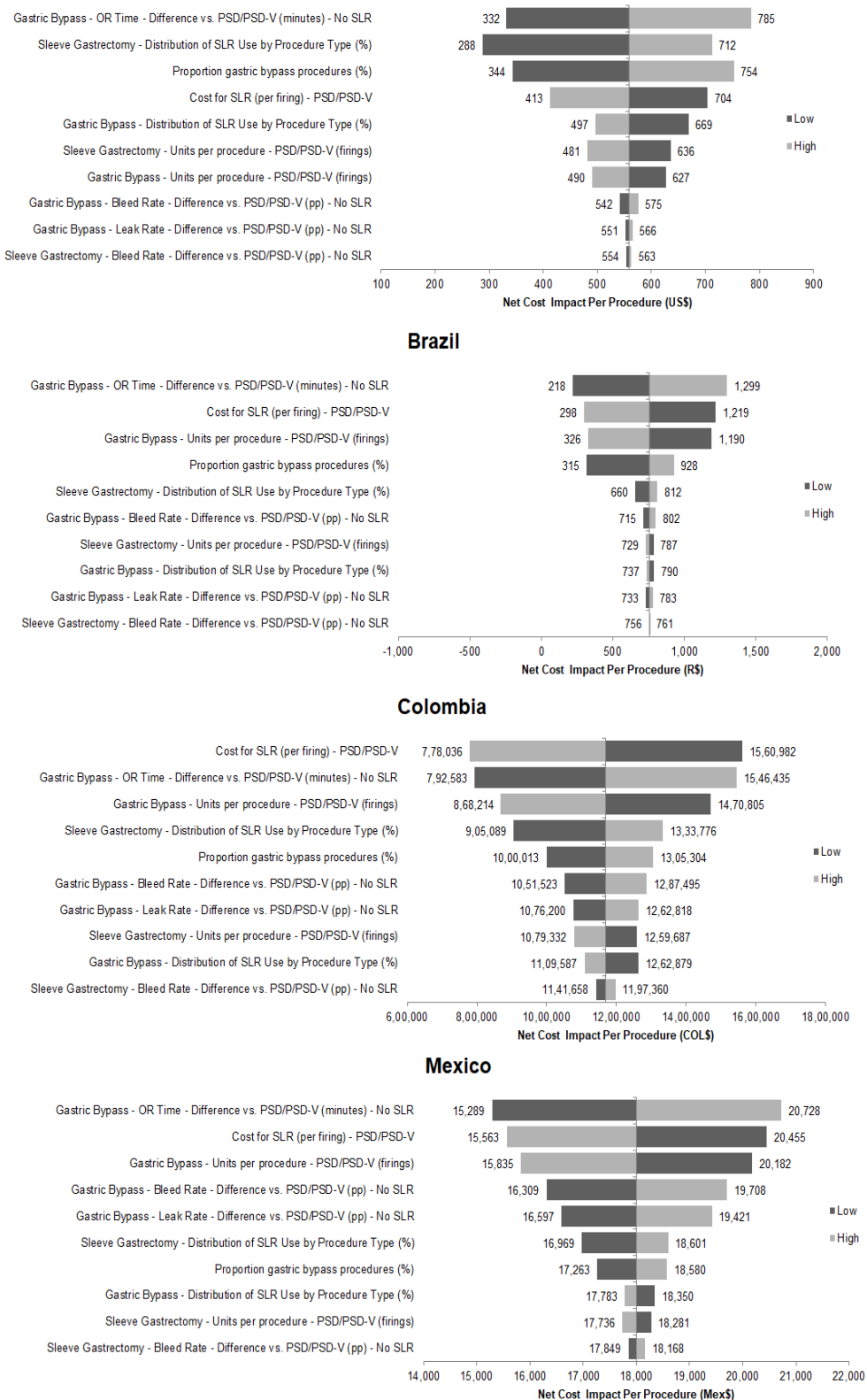

Figure 5: Estimated impact of using No SLR vs. PSD/PSD-V per procedure - One Way Sensitivity Analysis - Tornado Diagram. 


\section{Discussion}

Per Procedure Cost Impact of Choosing PSD/PSD-V for Gastric SLR: Per procedure, the largest cost savings in Latin American countries come from switching to PSD/PSD-V from oversewing due primarily to the long OR time associated with the oversewing method of SLR (US\$98, 95\% CI= -US\$188-US\$391; R $\$ 3,558,95 \%$ $\mathrm{CI}=\mathrm{R} \$ 2,199-\mathrm{R} \$ 5,047$; COL $\$ 2.42$ million, 95\% CI=COL $\$ 1.47$ millionCOL $\$ 3.51$ million; Mex $\$ 28,700,95 \%$ CI=Mex $\$ 21,592-\operatorname{Mex} \$ 37,049$ ) (Table 4).

\begin{tabular}{|c|c|c|c|c|}
\hline & United States & Brazil & Colombia & Mexico \\
\hline \multicolumn{5}{|l|}{ Seamguard vs. PSD/PSD-V } \\
\hline Complications Avoided & US $\$ 1,948$ & $\mathrm{R} \$ 1,151$ & $\operatorname{COL} \$ 4,441,587$ & Mex $\$ 53,589$ \\
\hline Operating Room Savings & US $\$ 2,307$ & $\mathrm{R} \$ 645$ & COL $\$ 662,552$ & Mex $\$ 3,650$ \\
\hline Product Cost Difference & - US\$1,682 & - $\mathrm{R} \$ 2,752$ & - COL $\$ 1,715,809$ & - Mex\$13,415 \\
\hline Net Impact & US $\$ 2,573$ & $\mathbf{0}$ & $\operatorname{COL} \$ 3,388,330$ & Mex $\$ 43,823$ \\
\hline \multicolumn{5}{|l|}{ Oversewing vs. PSD/PSD-V } \\
\hline Complications Avoided & US $\$ 256$ & $\mathrm{R} \$ 10,504$ & $\operatorname{COL} \$ 32,857,937$ & Mex $\$ 470,586$ \\
\hline Operating Room Savings & US $\$ 4,318$ & $\mathrm{R} \$ 139,954$ & COL $\$ 93,150,138$ & Mex\$693,586 \\
\hline Product Cost Difference & - US\$3,982 & - $\mathrm{R} \$ 33,939$ & - COL $\$ 41,065,917$ & - Mex\$204,114 \\
\hline Net Impact & US\$592 & $\mathrm{R} \$ 116,519$ & COL\$84,942,158 & Mex\$960,058 \\
\hline \multicolumn{5}{|l|}{ No SLR vs. PSD/PSD-V } \\
\hline Complications Avoided & US $\$ 6,546$ & $\mathrm{R} \$ 17,479$ & COL $\$ 54,597,785$ & Mex\$785,358 \\
\hline Operating Room Savings & US $\$ 49,575$ & $\mathrm{R} \$ 131,282$ & COL $\$ 83,034,735$ & Mex\$642,167 \\
\hline Product Cost Difference & - US $\$ 31,749$ & - $\mathrm{R} \$ 111,925$ & - COL\$86,155,406 & - Mex $\$ 577,374$ \\
\hline Net Impact & US $\$ 24,372$ & $R \$ 36,836$ & COL $\$ 51,477,113$ & Mex\$850,151 \\
\hline
\end{tabular}

Abbreviations: COL\$, Colombian Pesos; Mex\$, Mexican Pesos; PSD/PSD-V, Peri-Strips Dry/Peri-Strips Dry with Veritas; R\$, Brazilian Reals; US\$, United States dollars.

Using no SLR during gastric surgery leads to the highest rates of SLC and potential per procedure cost savings of PSD/ PSD-V in the US, Colombia, and Mexico despite higher product acquisition costs (US\$559, 95\% CI=US\$212-US\$949; R\$758, 95\% $\mathrm{CI}=-\mathrm{R} \$ 197-\mathrm{R} \$ 1,775$; COL $\$ 1.17$ million, 95\% CI=COL $\$ 0.42$ millionCOL $\$ 1.98$ million; Mex $\$ 18,009,95 \%$ CI=Mex $\$ 12,145-\operatorname{Mex} \$ 24,311$ ).

PSD/PSD-V provides cost savings over Seamguard in the US and Mexico despite similar product costs and OR time due to a significant reduction in SLC (US\$95, 95\% CI=-US\$186-US\$376; -R \$207, 95\% $\mathrm{CI}=-\mathrm{R} \$ 985-\mathrm{R} \$ 567$; COL $\$ 0.51$ million, 95\% CI=-COL\$0.27 millionCOL\$1.29 million; Mex\$8,382, 95\% CI=Mex\$3,762-Mex\$12,913).

Clinical Impact of Choosing PSD/PSD-V for Gastric SLR: In addition to a cost impact by utilizing PSD/PSD-V across all primary bariatric procedures, the number of complications would decrease by approximately $0.4,2.9,2.8$, and 2.8 complications per 100 procedures in the US, Brazil, Colombia, and Mexico, respectively.

Therefore, the use of PSD/PSD-V across all primary bariatric procedures is a dominant strategy (fewer complications, lower cost). Although the cost impact per procedure is not always significantly greater than zero (not favoring PSD/PSD-V), there is a cost effectiveness argument that could be developed based on the net cost impact and number of complications avoided.

Annual Cost Impact of Choosing PSD/PSD-V for Gastric SLR: The net annual cost savings of switching to PSD/PSD-V from other gastric SLR methods for a hospital performing 100 primary bariatric/metabolic procedures annually would be $\quad$ US\$27,537 $(95 \% \quad \mathrm{CI}=\mathrm{US} \$ 6,572-\mathrm{US} \$ 51,509), \quad \mathrm{R} \$ 152,399$ (95\% CI=R $\$ 66,764-\mathrm{R} \$ 253,925)$, COL $\$ 139.81$ million $(95 \%$ CI=COL $\$ 72.48$ million-COL $\$ 220.95$ million), and Mex $\$ 1,854,032$ (95\% CI=Mex\$1,256,153-Mex\$2,560,758). These results are robust given that the lower bound of the $95 \%$ confidence interval in each country still shows cost savings across 5000 iterations.

Limitations: In the case of the distribution of SLR methods across procedures, country-specific inputs were not available. Variability in this input was allowed in both the one-way sensitivity analysis and PSA, but the relative influence of annual net cost savings from primary bariatric/metabolic procedures was not large. Only the US distribution of SLR use for gastric bypass was a top-5 influencer of annual net cost savings for primary metabolic procedures. Based on our modelled results, more gastric bypass and sleeve gastrectomy procedures being performed without SLR or using the Seamguard and oversewing methods would lead to greater benefit of PSD/PSD-V in terms of patient outcomes and a low to positive cost impact in favor of PSD/PSD-V.

Additionally, given lack of data, US product costs for PSD/PSD-V, Seamguard and oversewing were converted to local currencies and used as a proxy for Brazil, Colombia and Mexico. The costs of PSD/ PSD-V and Seamguard were both influential in sensitivity analysis. In the US, these costs per firing for these products are roughly equivalent. Therefore, a hospital administrator is acquiring both products for different prices, the results of this research may not hold (Appendix 1-3). 


\section{Conclusion}

A high volume hospital preforming 100 primary bariatric/ metabolic procedures per year could potentially realize cost savings of US $\$ 27,537$ (95\% CI=US $\$ 6,572$-US $\$ 51,509$ ), R $\$ 152,399$ (95\% CI=R \$66,764-R \$253,925), COL\$139.81 million (95\% CI=COL\$72.48 million-COL $\$ 220.95$ million), and Mex $\$ 1,854,032$ (95\% CI=Mex $\$ 1,256,153-M e x \$ 2,560,758)$ and in the US, Brazil, Colombia, and, Mexico, respectively, by using PSD/PSD-V rather than other SLR choices. Despite the acquisition cost of PSD/PSD-V, SLR using PSD/PSD-V demonstrates cost savings associated with reductions in complications (i.e., leaks and bleeds) and OR time.

\section{Acknowledgments}

None

\section{Conflict of Interest}

This study was funded by Baxter Healthcare Corporation MGR and MAR are paid employees and stockholders at Baxter. AG is a surgeon at the Anne Arundel Medical Center and was retained for this work. BB and JE are employees at Stratevi, which was retained for this work.

\section{References}

1. NCD Risk Factor Collaboration (NCD-RisC) (2017) Worldwide trends in body-mass index, underweight, overweight, and obesity from 1975 to 2016: A pooled analysis of 2416 population-based measurement studies in 128.9 million children, adolescents, and adults. Lancet 390(10113): 2627-2642.

2. Robert B Lim (2018) Bariatric procedures for the management of severe obesity. UpToDate.

3. Ali M, El Chaar M, Ghiassi S, Rogers AM, American Society for Metabolic and Bariatric Surgery Clinical Issues Committee (2017) American society for metabolic and bariatric surgery updated position statement on sleeve gastrectomy as a bariatric procedure. Surg Obes Relat Dis 13(10): 1652-1657.

4. Iannelli A, Treacy P, Sebastianelli L, Schiavo L, Martini F (2019) Perioperative complications of sleeve gastrectomy: Review of the literature. J Minim Access Surg 15(1): 1-7.

5. Himpens J, Ramos A, Welbourn R, et al. (2018) Fourth ifso global registry report. The International Federation for the Surgery of Obesity and Metabolic Disorders. Oxfordshire UK, 2018.

6. Shikora SA, Mahoney CB (2015) Clinical benefit of gastric staple line reinforcement (SLR) in gastrointestinal surgery: A meta-analysis. Obes Surg 25(7): 1133-1141.

7. Zambelli Weiner A, Brooks E, Brolin R, Eric S Bour (2013) Total charges for post-operative leak following laparoscopic sleeve gastrectomy. Obesity Week Atlanta GA.

8. Bransen J, Gilissen LP, van Rutte PW, Nienhuijs SW (2015) Costs of leaks and bleeding after sleeve gastrectomies. Obesity surgery 25(10): 1767 1771

9. Nedelcu M, Manos T, Gagner M, Eddbali I, Ahmed A (2017) Cost analysis of leak after sleeve gastrectomy. Surgical endoscopy 31(11): 4446-4450.

10. Ahmed A, Adamo M, Balchandra S (2015) The real costs of treating early post-operative leaks following sleeve gastrectomy procedures. SPRINGER p. 45.
11. Haskins 0 (2017) Are all staple line reinforcement materials equal? Bariatric News.

12. Peri-Strips Dry with Veritas (2018) Instructions for use. Baxter Healthcare Corporation, Deerfield, Ireland.

13. Peri-Strips Dry (2018) Instructions for use. Baxter Healthcare Corporation, Deerfield, Ireland.

14. Seamguard (2012) Instructions for use. Flagstaff, AZ: WL Gore \& Assiciates.

15. Higa K, Himpens J, Welbourn R (2017) Third ifso global registry report. The International Federation for the Surgery of Obesity and Metabolic Disorders. Oxfordshire, Uk.

16. Ministério da Saúde (2017) Cirurgia bariátrica por laparoscopia. Relatorio de Recomendacao.

17. IMS. Hospital supply index (hsi). 2012-2015; Danbury (CT): IMS Health Inc.

18. Premier. Us data base. 2012-2015; Unpublished raw data.

19. Metabolic and Bariatric Surgery Accreditation and Quality Improvement Program (MBSAQIP). Us database. 2015-2018.

20. Guilbert L, Joo P, Ortiz C, Sepúlveda E, Alabi F, et al. (2019) Safety and efficacy of bariatric surgery in mexico: A detailed analysis of 500 surgeries performed at a high-volume center. Rev Gastroenterol Mex 84(3): 296-302.

21. Sepúlveda M, Astorga C, Hermosilla JP, Alamo M (2017) Staple line reinforcement in laparoscopic sleevegastrectomy: Experience in 1023 consecutive cases. Obesity surgery 27(6): 1474-1480.

22. Premier. Us data base. 2017; Unpublished raw data.

23. Mexican Social Security Institute (IMSS) (2016) Inpatient hospital drg's book drg 619.

24. Mexican Social Security Institute (IMSS) (2016) Inpatient hospital drg's book drg 620 .

25. Sajid MS, Khatri K, Singh K, Sayegh M (2011) Use of staple-line reinforcement in laparoscopic gastric bypass surgery: a meta-analysis. Surg Endosc 25(9): 2884-2891.

26. Gentileschi P, Camperchioli I, DUgo S, Benavoli D, Gaspari AL (2012) Staple-line reinforcement during laparoscopic sleeve gastrectomy using three different techniques: A randomized trial. Surg Endosc 26(9): 2623-2629.

27. Shah SS, Todkar JS, Shah PS (2014) Buttressing the staple line: A randomized comparison between staple-line reinforcement versus no reinforcement during sleeve gastrectomy. Obes Surg 24(12): 2014-2020.

28. IMS. Hospital supply index (hsi). 2018; Danbury (CT): IMS Health Inc.

29. International Monetary Fund. Consumer price index, all items. IMF Data 2018.

30. International Monetary Fund. Exchange rate, sdrs per currency unit. IMF Data. 2018.

31. Barba EJR, Suárez MEC (2015) Monterrosa EdCS. Transfusión de paquete globular. Del beneficio clínico real a la inadecuada prescripción. Revista Latinoamericana de Patología Clínica y Medicina de Laboratorio 62(1): 46-54

32. Mexican Social Security Institute (IMSS) (2017) Inpatient hospital drg's book average drg's $30,455,458,460,473,491$ plus surgical procedure in a 3 rd hospital level.

33. Chatterjee A, Payette MJ, Demas CP, Finlayson SR (2009) Opportunity cost: A systematic application to surgery. Surgery 146(1): 18-22. 